\title{
Why Quasi-Stationary Refraction Blocks but not Multipath in GPS
}

\section{VC}

\section{Perović G*}

Department of Geodesy and Geoinformatics, Faculty of Civil Engineering, University of Belgrade, Serbia

*Corresponding author: Gligorije Perovic, Department of Geodesy and Geoinformatics, Faculty of Civil Engineering, University of Belgrade, Serbia, Tel: +381 11 3218539; Email:

\section{Mini Review}

Volume 4 Issue 2

Received Date: August 08, 2020

Published Date: August 27, 2020 perg@grf.bg.ac.rs

\section{Abstract}

The paper presents that quasi-stationary refraction blocks have been studied, ie. know, before the advent of GPS, as well as that GPS measurement errors caused by these blocks make up one of the main components of GPS measurement variance, while errors caused by multipat do not belong to the main components of variance.

Keywords: GPS; Quasi-stationary refraction blocks; PERG2FH ANOVA; FLI-MAP

\section{Mini Review}

Information on quasi-stationary refraction blocks started to be completed very long ago - with comencements of precise horizontal angle measurements, i. e. in National trigonometric first-order networks. More correctly, with comencements of detailed error investigation in those networks. So, for instance, Russian geodesists already 150 years ago disccovered that in the atmospheric parts near the ground there are atmospheric layers (current name: blocks) which are unstable - vacillate - and influence the accuracy of precise measurements of horizontal angles by limiting it.

These results of the Russian geodesists were immediately after 1917 transferred into Serbia. So, we students in the framework of our ten-day practice of precise measuring of horizontal angles in 1965 were learning and convincing about the existence of quasi-stationary (lateral) refraction blocks and their mode of action on horizontal angle measurement errors. The measurements were performed in stable conditions, and the influence of quasi-stationary (lateral) refraction blocks reflected in the following way: In the first time interval of angle measuring of duration of, say, one hour, the values are within 2-3", then there is another time interval, of say about 40 minutes, where the values are again within 2-3", but the discrepancy in angle mean values from these two time intervals is high, even 7" and more. In the posterior measurements the situation is repeated followed by similar results.

Physics of the atmosphere has been thoroughly studied in the world. Among others, extraordinarily good and detailed studies of atmosphere physics has been carried out by Russian scientists with a special group of researchers who for 13 years over a special geopolygone in the Carpathian mountain massif performed measurements. Part of their results was published in 1969 in monograph [1].

In the physical sense a quasi-stationary light refraction block is defined as a part of atmosphere along the ray on which due to the external conditions influence stable gradients of tempereture and air humidity are formed [1]. Quasi-stationary refraction blocks exist at every atmospheric point and at any time, so that the mentioned group of Russian scientists use one of the accurate ways to determine the refraction vector by means of meteorological measurements, the way of its obtaining by using the measured gradients of tempereture, huminidity and air pressure [1]. These 
determinations were done for terrestrial measurements, for one up to artificial satellites and for astronomical measurements.

Thus all of this had been known well before GPS measurements, when the multipath did not exist!

From the beginning of extensive GPS application in geodesy - from 2000 - I have for more than 10 years studied the sources and structure of GPS measurements errors, where I noticed that the quasi-stationary refraction blocks appear as error sources limiting the accuracy of GPS measurements. According to my experience stationarity of these blocks lasts from 20 minutes to 2 hours, but Yunoshev [1] gives an example of duration of these blocks over night attaining 3 hours. So, in 2004 I used the knowledge concerning the influence of quasi-stationary refraction blocks in order to improve the accuracy of determining asphalt road points heights obtained by application of FLI-MAP technology [2,3]. The accuracy of asphalt road points heights determined by use of the standard FLI-MAP procedure was $5-8 \mathrm{~cm}$ depending on the helicopter flight height. For this purpose the road Bačka Palanka - Sombor 80 km long was surveyed, and for the purpose of more accurate determination for point heights along the road reference height points were regularly spaced $5 \mathrm{~km}$ mutually distant. For this $5 \mathrm{~km}$ distance at a speed of $50 \mathrm{~km} / \mathrm{h}$ the helicopter needs 6 minutes. Therefore, the stationarity of refraction blocks, of more than 20 minutes duration, led to errors of road point FLI-MAP heights decreased to $2 \mathrm{~cm}$ (standard).

GPS errors have been in detail studied by many authors [4-11]. The multipath has been also studied in detailed $[4,6,12-15]$, and it leads to high errors in GPS measurements, i. e. there are outliers. Because of satellite motion the multipath duration is very short, of order of 2-4 minutes so that these errors are not numerous. They are easily noticed on a plot and are easily detected by means of any gross error test. On the diagram of three-day measuring results, Figure 1 , on the base vector Kragujevac - Batočina (KB) in Serbia multipath peaks are clearly seen as well as irregular effects of quasi-stationary refraction blocks. Thus, multipath is clearly seen for abscisae: $1300,4150,7850$ i 8050 ; but the results for 2700,5600 i 8350 can be also added.

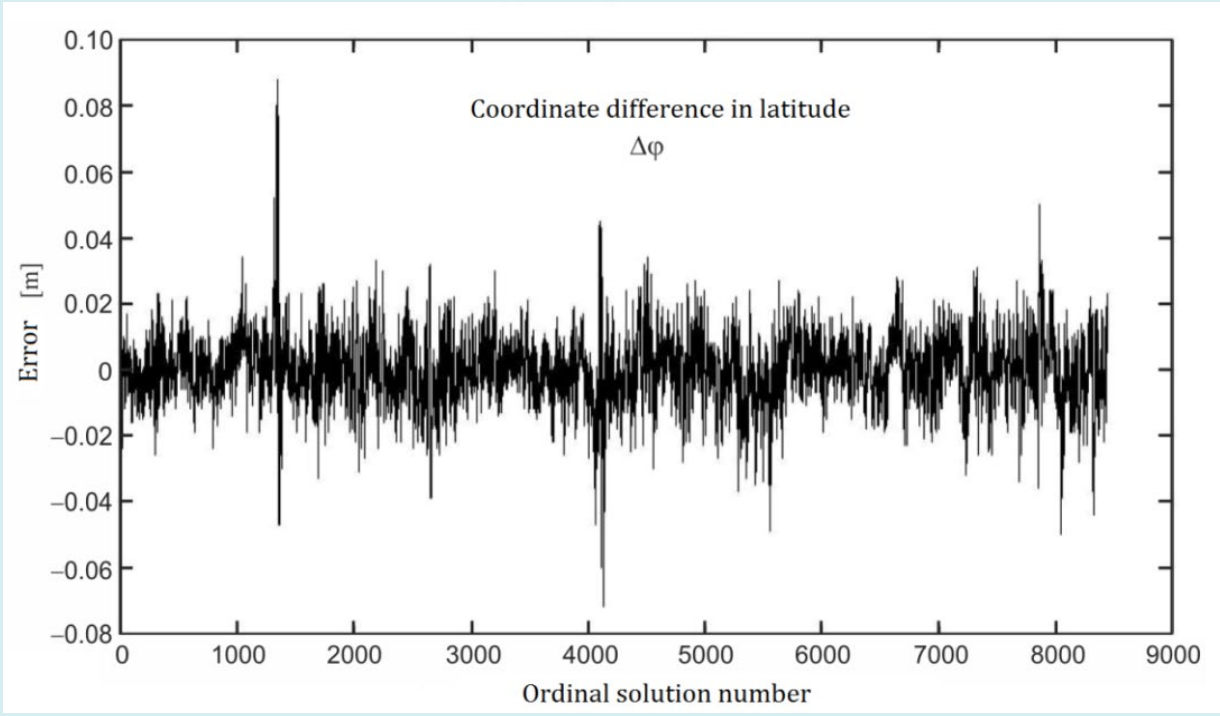

Figure 1: Three-day measuring results on the base vector Kragujevac - Batočina (KB) in Serbia multipath peaks are clearly seen as well as irregular effects of quasi-stationary refraction blocks.

Studying the GPS measurement errors I found a key by which I unlocked the random process of GPS measurements and I saw its structure which with its mathematical idealization is presented in Figure 2. So I concluded that the error structure of GPS measurements belong to the two-way hierarchical (nested) clasification with random effects.

$\alpha$ - tropo + iono effects, $\beta$ - quasi-stationary refraction blocks effekts, $\varepsilon=$ purely random error: 


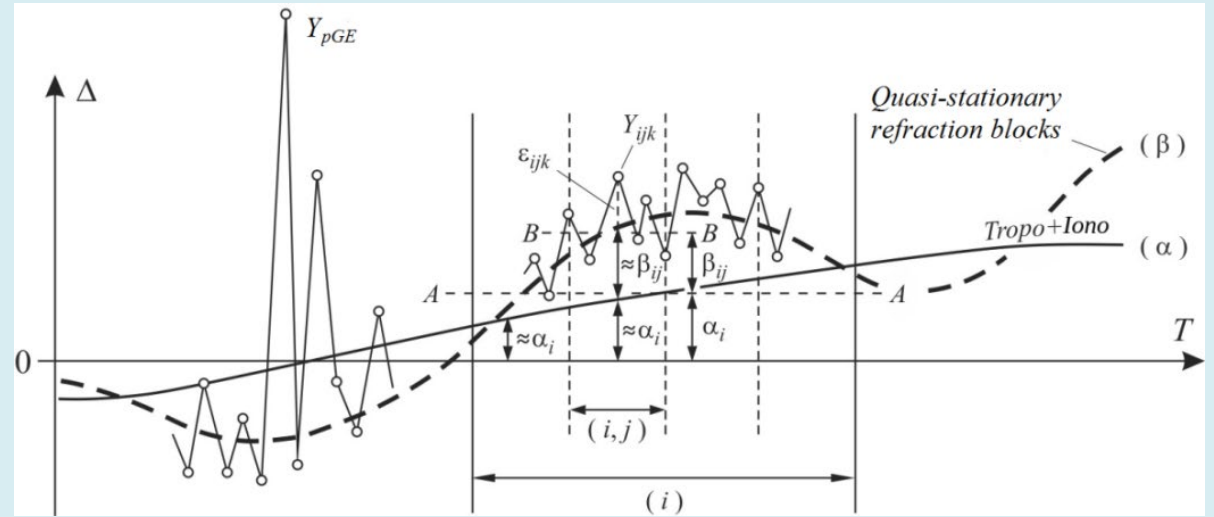

Figure 2: Presentation of errors of GPS measurements (without constant systematic error) by using 2-way hierarchical classification in PERG2FH method: Total error $\Delta_{i j k}=\alpha_{i}+\beta_{i j}+\varepsilon_{i j k} ; \alpha=$ tropo + iono, $\beta=$ quasi-stationary refraction blocks, $\varepsilon=$ purely random error, $Y_{p G E}=$ outlier (result with Gross Error), $A-A$ =horizontal, $B-B=$ horizontal; Intervals: $(i)-$ for $\alpha$ ,$(i, j)-$ for $\beta$.

$$
\begin{gathered}
Y_{i j k}=\mu+\alpha_{i}+\beta_{i j}+\varepsilon_{i j k},(1) \\
i=1, \cdots, a, j=1,2, \cdots, b_{i}, k=1,2, \cdots, n_{i j} ;(2) \\
N_{i}=\sum_{j=1}^{b_{i}} n_{i j}=n_{i .}, B=\sum_{i=1}^{a} b_{i}=b ., N=\sum_{i=1}^{a} \sum_{j=1}^{b_{i}} n_{i j} \\
n_{i j} \geq 2, b_{i} \geq 2, a \geq 2 \text {. (4) }
\end{gathered}
$$

with the stochastic model:

$$
\begin{gathered}
\alpha_{i} \square N\left[0, \sigma_{\alpha}^{2}\right], \forall i ; \beta_{i j} \square N\left[0, \sigma_{\beta}^{2}\right], \forall i, j ; \\
\varepsilon_{i j k} \square N\left[0, \sigma^{2}\right], \forall i, j, k,(5) \\
C\left[\alpha_{i}, \beta_{i j}\right]=\mathrm{C}\left[\alpha_{i}, \varepsilon_{i j k}\right]=\mathrm{C}\left[\beta_{i j}, \varepsilon_{i j k}\right]=0, ; \forall i, j, k, \\
\mathrm{C}\left[\varepsilon_{i j k}, \varepsilon_{i^{\prime} j^{\prime} k^{\prime}}\right]=0, \forall k \neq k^{\prime},(6)
\end{gathered}
$$

where $\mu=\mathrm{E}\left[Y_{i j k}\right], \forall i, j, k$ and $\mathrm{C}[u, v]$ is the covariance operator between $u$ and $v$.

The article about VC in GPS measurements published in 2016 [16] from which we are presenting only the results of the analysis. PERG2FH method of VC analysis in GPS measurements was applied, where four base vectors were processed:

1. base vector Bor-Kruševac (BK)

magnitude $80 \mathrm{~km}$; 1 day measurements,

2. base vector Bar-Podgorica (BP) magnitude $40 \mathrm{~km}$; 3 years measurements,

3. base vector Kragujevac-Batočina (KB) magnitude $20 \mathrm{~km} ; 3$ day measurements, $\mathrm{i}$
4. base vector Beograd (Bg)

magnitude $2 \mathrm{~km}$; 3 day measurements.

The measurements for for two-way hierarchical analysis are divided into intervals: $I_{\alpha}$ - for factor $\alpha$ and $I_{\beta}$-for factor $\beta$.

Simultaneously with the ANOVA analysis gross error test are performed, where in Table 1 the results for vector BK with $I_{\alpha}=6 \mathrm{~h}$ are given.

The gross error tests were applied to deviations of single results from the mean value (local tests) with the significance level $\alpha_{0}=0.001$. The number of results removed for BK vector with $I_{\beta}=7.5 \mathrm{~min}$ and $I_{\alpha}=6 \mathrm{~h}$ is given in Table 1 .

\begin{tabular}{|c|c|c|c|}
\hline & $\Delta \lambda$ & $\Delta \varphi$ & $\Delta h$ \\
\hline Test for $Y_{i j k}:$ & $21 ;[0.7 \%]$ & $35 ;[1.2 \%]$ & $43 ;[1.5 \%]$ \\
\hline Test for $\bar{Y}_{i j}:$ & - & - & 1 \\
\hline Test for $\bar{Y}_{i}:$ & - & - & - \\
\hline$N:$ & 2855 & 2841 & 2833 \\
\hline$B:$ & 192 & 192 & 191 \\
\hline$a:$ & 4 & 4 & 4 \\
\hline
\end{tabular}

Table 1: The results for vector BK with $I_{\alpha}=6 \mathrm{~h}$.

PERG2FH ANOVA analysis of GPS VC is given in Tables 2-4. The following designations are used:

$m_{\varepsilon_{2}}^{2}$ - estimate for $\sigma_{\varepsilon}^{2}, m_{\beta}^{2}$ - estimate for $\sigma_{\beta}^{2}, m_{\alpha}^{2}$ - estimate for $\sigma_{\alpha}^{2}, m_{\Delta}^{2}$ - estimate for $\sigma_{\Delta}^{2} ; f_{i}$ - d. f. for $i=\varepsilon, \beta, \alpha$. 


\section{Physical Science \& Biophysics Journal}

\begin{tabular}{|c|c|c|c|c|c|c|c|c|}
\hline & \multicolumn{2}{|c|}{ For $\Delta \lambda:$} & \multicolumn{2}{c|}{ For $\Delta \varphi:$} & \multicolumn{2}{c|}{ For $\Delta h:$} \\
\hline$I_{\alpha}:$ & $6 \mathrm{~h}$ & $3 \mathrm{~h}$ & & $6 \mathrm{~h}$ & $3 \mathrm{~h}$ & \multicolumn{2}{|c|}{$6 \mathrm{~h}$} & $3 \mathrm{~h}$ \\
\hline$m_{\varepsilon}[\mathrm{mm}]$ & 4.5 & 5.6 & & 5.6 & 5.6 & & 4.5 & 5.6 \\
\hline$m_{\beta}[\mathrm{mm}]$ & 4.9 & 4.7 & & 6.2 & 5.9 & & 13.5 & 12.0 \\
\hline$m_{\alpha}[\mathrm{mm}]$ & 2.2 & 2.4 & & 3.0 & 3.5 & & 11.3 & 11.6 \\
\hline$m_{\Delta}[\mathrm{mm}]$ & 8.9 & 7.7 & & 7.0 & 8.9 & & 18.2 & 17.6 \\
\hline$f_{\varepsilon}:$ & 2663 & 2663 & & 2649 & 2649 & & 2642 & 2601 \\
\hline$f_{\beta}{ }^{1}:$ & 128.63 & 152.76 & & 169.02 & 163.62 & & 184.28 & 174.79 \\
\hline$f_{\alpha}{ }^{1}:$ & 2.42 & 5.11 & & 2.51 & 5.50 & & 2.82 & 6.40 \\
\hline
\end{tabular}

Table 2: PERG2FH ANOVA results survey for BK vector ( $80 \mathrm{~km})$ at $I_{\alpha}=6 \mathrm{~h}, 3 \mathrm{~h} ; I_{\beta}=7.5 \mathrm{~min}$.

In Figure 3 for the BP vector (40km) the PERG2FH ANOVA VC analysis results (from [11]) are presented.

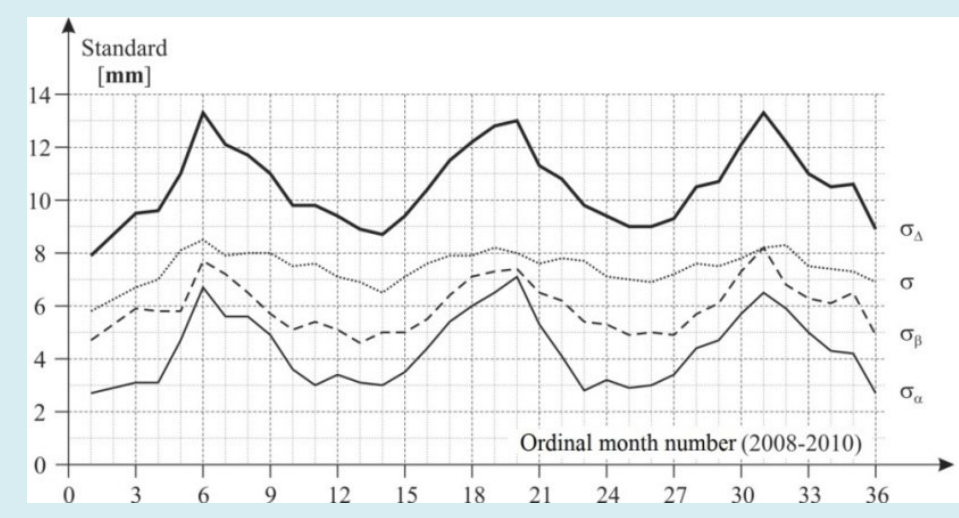

Figure 3: Plots of standards $\sigma=\sigma_{a}, \sigma_{\beta}$ and $\sigma_{a}$, and total error $\sigma_{\Delta}$ for coordinate difference $\Delta \varphi$ of base GPS vector BP $(40 \mathrm{~km})$ obtained following PERG2FH ANOVA during three years, $2008-2010 ;\left(I_{\alpha}=2 \mathrm{~h} ; I_{\beta}=7.5 \mathrm{~min}\right.$.).

\begin{tabular}{|c|c|c|c|c|c|c|c|}
\hline & & \multicolumn{2}{|c|}{ For $\Delta \lambda$} & \multicolumn{2}{c|}{ For $\Delta \varphi$} & \multicolumn{2}{c|}{ For $\Delta h$} \\
\hline$I_{\alpha}:$ & & $6 \mathrm{~h}$ & $2 \mathrm{~h}$ & $6 \mathrm{~h}$ & $2 \mathrm{~h}$ & $6 \mathrm{~h}$ & $2 \mathrm{~h}$ \\
\hline$m_{\varepsilon}[\mathrm{mm}]$ & & 5.5 & 5.5 & 8.0 & 8.0 & 14.3 & 14.3 \\
\hline$m_{\beta}[\mathrm{mm}]$ & & 5.2 & 4.7 & 6.2 & 5.8 & 14.8 & 12.3 \\
\hline$m_{\alpha}[\mathrm{mm}]$ & & 3.4 & 2.7 & 2.3 & 3.1 & 16.4 & 12.1 \\
\hline$m_{\wedge}[\mathrm{mm}]$ & & 8.3 & 7.7 & 10.4 & 10.4 & 26.3 & 22.4 \\
\hline
\end{tabular}

Table 3: PERG2FH ANOVA results for $\mathrm{KB}$ vectror $(20 \mathrm{~km})$ for $I_{\beta}=7.5 \mathrm{~min}$.

\begin{tabular}{|cc|c|c|c|c|c|}
\hline & \multicolumn{2}{c|}{ For $\Delta \lambda$} & \multicolumn{2}{c|}{ For $\Delta \varphi$} & \multicolumn{3}{c|}{ For $\Delta h$} \\
\hline$I_{\alpha}:$ & $6 \mathrm{~h}$ & $2 \mathrm{~h}$ & $6 \mathrm{~h}$ & $2 \mathrm{~h}$ & $6 \mathrm{~h}$ & $2 \mathrm{~h}$ \\
\hline$m_{s}[\mathrm{~mm}]$ & 2.2 & 2.2 & 3.3 & 3.3 & 5.4 & 5.4 \\
\hline$m_{\beta}[\mathrm{mm}]$ & 2.1 & 1.2 & 3.2 & 2.9 & 5.5 & 5.1 \\
\hline$m_{\alpha}[\mathrm{mm}]$ & 0.74 & 1.1 & 0.68 & 1.4 & 1.0 & 2.1 \\
\hline$m_{\wedge}[\mathrm{mm}]$ & 3.1 & 3.1 & 4.7 & 4.6 & 7.8 & 7.7 \\
\hline
\end{tabular}

Table 4: PERG2FH ANOVA results for Bg vectror $(2 \mathrm{~km})$ for $I_{\beta}=7.5 \mathrm{~min}$.

1 In 2008 I derived the formulae for these degrees of freedom. 
The correlation of variance components does not exist because the maximum correlation coefficients are: $\max |r|=\mid r($ alpha,beta $) \mid=0.04 \quad$ - for BK vector $(80 \mathrm{~km})$, $\max |r|=\mid r($ alpha,beta $) \mid=0.03 \quad$ - for BP vector $(40 \mathrm{~km})$, $\max |r|=\mid r($ alpha,beta $) \mid=0.02$ - for KB vector $(20 \mathrm{~km})$, $\max |r|=\mid r($ alpha,beta $) \mid=0.03 \quad$ - for Bg vector $(2 \mathrm{~km})$, thus confirming assumptions (6).

\section{Conclusion}

In conclusion, we emphasize that the presented results confirmed the assumptions about quasi-stationary refraction blocks, as well as that the validity of the model that the error structure of GPS measurements belongs to the two-way hierarchical (nested) classification with random effects.

\section{References}

1. Yunoshev LS (1969) Lateral refraction in angle measurements, Monograph. Nedra, Moscow, Russia.

2. Perović G (2004a) PERG Method of Increasing the Absolute Vertical Accuracy of the Laser Scanned points Obtained by FLI-MAP System Applied on Asphalt Roads. FUGRO, Leidschendam, Netherlands.

3. Perović G, Nestorov I, Beljin J, Gligorić M, (2004b) PERG method of Increasing the Absolute Vertical Accuracy of the Laser Scanned Points Obtained by FLI-MAP System Applied on Asfalt Roads. Intergeoeast, Belgrade.

4. Langley RB (1998) Propagation of the GPS Signals. In: Teunissen PJG, Kleusberg A (Eds.), GPS for Geodesy. 2nd (Edn.), Springer-Ferlag, Berlin-Heidelberg.

5. Teunissen PJG, Kleusberg A (1998) GPS observation equations and positioning concepts. In: Teunissen PJG, Kleusberg A (Eds.), GPS for Geodesy. 2nd (Edn.), Springer-Ferlag, Berlin-Heidelberg, pp: 187-229.

6. Blagojević D (2003) Possibilities of GPS establishment of local reference geodetic frame. Doctoral Dissertation,
Faculty of Civil Engineering, Department of Geodesy, Belgrade.

7. Perović G (2008) The PERG method of noise dispersion estimation in relative GPS positioning. Intergaoeast, Belgrade.

8. Perović G (2009) Principle of Determining the Influences of Time Variable Errors on the Baseline Vectors. International Conference on Geodesy, Cartography and Cadastre in the 21th Century Moscow State University of Geodesy and Cartography, Moscow, Russia.

9. Hofmann-Wellenhof B, Lichtenegger H, Collins J (2012) Global Positioning System: Theory and Practice. 5th (Edn.), Springer, pp: 382.

10. Perović G (2017) Precise geodetic measurements, Monograph 2. AGM knjiga, Belgrade.

11. Andjić D (2019) Estimation of Time-Variable Error Variance Components in GPS Determination of Coordinates, Doctoral Dissertation. Faculty of Civil Engineering, Department of Geodesy, Belgrade.

12. Townsend BR, Fenton PC (1994) A Practical Approach to the Reduction of Pseudorange Multipath Errors in a Ll GPS Receiver. ION GPS-94, Salt Lake City, USA, pp: 43148.

13. Rao BRK, Sarma AD, Kumar YR (2006) Technique to reduce multipath GPS signals. Current Science 90(2): 2007-211.

14. Mehaffey J (2016) Multipath Errors.

15. Vázquez BGE, Grejner-Brzeziska A (2012) A case study for Pseudorange multipath estimation and analysis: TAMDEF GPS network. Geofís Intl 51(1).

16. Perović G (2016) Variance Components Analysis in GPS Measurements. Imperial Journal of Interdisciplinary Research 2(11). 